\title{
Mitogen-Activated Protein Kinase Kinase
}

National Cancer Institute

\section{Source}

National Cancer Institute. Mitogen-Activated Protein Kinase Kinase. NCI Thesaurus.

Code C105947.

A family of dual-specific protein kinases that are activated through phosphorylation by mitogen-activated protein kinase kinase kinase (MAP3K, MAPKKK) proteins and can subsequently phosphorylate and activate mitogen-activated protein kinases (MAPK). 